University of Wollongong

Research Online

Faculty of Informatics - Papers (Archive)

Faculty of Engineering and Information

Sciences

$1-1-2010$

\title{
Adaptive regularization for image restoration using a variational inequality approach
}

Matthew Kitchener

University of Wollongong, mak55@uow.edu.au

Abdesselam Bouzerdoum

University of Wollongong, bouzer@uow.edu.au

Son Lam Phung

University of Wollongong, phung@uow.edu.au

Follow this and additional works at: https://ro.uow.edu.au/infopapers

Part of the Physical Sciences and Mathematics Commons

\section{Recommended Citation}

Kitchener, Matthew; Bouzerdoum, Abdesselam; and Phung, Son Lam: Adaptive regularization for image restoration using a variational inequality approach 2010, 2513-2516.

https://ro.uow.edu.au/infopapers/835

Research Online is the open access institutional repository for the University of Wollongong. For further information contact the UOW Library: research-pubs@uow.edu.au 


\title{
Adaptive regularization for image restoration using a variational inequality approach
}

\begin{abstract}
In this paper, a generalized image restoration method is formulated as a variational inequality problem, whose solution is obtained using a dynamic system approach. In this method, the restored image and the regularization parameter are obtained simultaneously. In particular, the optimum regularization parameter is determined adaptively, depending on noise and image content. The restoration problem is presented in a generalized form so that it maybe be implemented using different norms; only L1 and L2 norms have been implemented in this paper. A comparison based on experimental results shows that the proposed method achieves comparable if not better performance as some of the existing state-of-the-art techniques.
\end{abstract}

\section{Keywords}

restoration, variational, inequality, adaptive, approach, regularization, image

\section{Disciplines}

Physical Sciences and Mathematics

\section{Publication Details}

M. Kitchener, A. Bouzerdoum \& S. Phung, "Adaptive regularization for image restoration using a variational inequality approach," in ICIP 2010: IEEE 17th International Conference on Image Processing, 2010, pp. 2513-2516. 


\title{
ADAPTIVE REGULARIZATION FOR IMAGE RESTORATION USING A VARIATIONAL INEQUALITY APPROACH
}

\author{
M. A. Kitchener, A. Bouzerdoum, IEEE Senior Member, and S. L. Phung, IEEE Member \\ School of Electrical, Computer and Telecommunications Engineering \\ University of Wollongong \\ Wollongong, NSW 2522, Australia
}

\begin{abstract}
In this paper, a generalized image restoration method is formulated as a variational inequality problem, whose solution is obtained using a dynamic system approach. In this method, the restored image and the regularization parameter are obtained simultaneously. In particular, the optimum regularization parameter is determined adaptively, depending on noise and image content. The restoration problem is presented in a generalized form so that it maybe be implemented using different norms; only $L_{1}$ and $L_{2}$ norms have been implemented in this paper. A comparison based on experimental results shows that the proposed method achieves comparable if not better performance as some of the existing state-of-theart techniques.
\end{abstract}

\section{INTRODUCTION}

Image restoration techniques aim to remove the distortions and noise in an image, in order to produce a more accurate representation of an imaged scene. This is generally achieved by implementing some kind of inverse procedure, which attempts to reverse the degradations that occur during image capture. Regularized image restoration techniques are an extension of these inverse procedures, and are used to deal with the ill-posed or ill-conditioned nature of the image restoration problem.

One of the most popular regularization approaches is Tikhonov Regularization, in which $\boldsymbol{f}$ is found by minimizing $\|\boldsymbol{g}-H \boldsymbol{f}\|_{2}^{2}+\lambda\|R \boldsymbol{f}\|_{2}^{2}$, with $\lambda$ being chosen to satisfy some other criteria, for example the maximum likelyhood. In some cases $\lambda$ is simply chosen in an ad-hoc manner.

One of the main problems associated with regularized image restoration, however, is finding a value for the regularization parameter, $\lambda$, that achieves an optimal compromise between image fidelity(where the edges are preserved), and noise removal. There are many mathematical approaches, both stochastic and deterministic, for solving the regularization problem, all of which attempt to use a priori information

E-mails: mak55, a.bouzerdoum,phung@uow.edu.au. in order to reconstruct the image. Bayesian methods are particulary popular and are presented in [1].

In this paper, we use a general variational inequality approach to solve the image restoration problem. Furthermore, the solution of the variational inequality problem is formulated as a dynamic system. It is recognized in [2] that one of the difficulties associated with the Total Variations approach is that the exact Lagrange parameter $\lambda$ is a computationally intensive number to determine and as such the majority of TV approaches select the value of $\lambda$ in an adhoc manner [2][3][4][5]. The proposed method finds the regularization parameter automatically by solving a general convex constrained optimization problem.

In this paper we use the $L_{1}$ and $L_{2}$ norms in the constraint to deal with zero mean gaussian noise. It is possible, however, to use the same approach with different norms, to deal with different types of noise. There are a number of papers that detail the benefits of using differing norms to solve the image restoration problem, a particularly good example can be found in [4]. In [6] the $L_{1}$ norm is used to deal with salt and pepper noise and in [7] the so-called "Least Mean Forth" is used to deal with sub-Gaussian distributions.

Another of the main contributions of this paper is in showing how statistical noise estimates, such as wavelet denoising, can be used to calculate an adaptive value for $\lambda$ accounting for different images, noise variances, and blurs.

\section{PROBLEM FORMULATION}

Consider a lexicographically ordered vector $\boldsymbol{g}$ of length $n=$ $l \times m$, representing an $l$ by $m$ noisy blurred image:

$$
\boldsymbol{g}=H \boldsymbol{f}_{o}+\boldsymbol{v}
$$

where $H$ is the blur matrix, $f_{o}$ is the original image we seek to restore, and $v$ is the additive noise vector. Here the image restoration problem is formulated in a general form as

$$
\begin{aligned}
\text { minimize }_{\boldsymbol{f}} & \|R \boldsymbol{f}\|_{k_{1}}^{k_{1}} \\
\text { subject to } & \|H \boldsymbol{f}-\boldsymbol{g}\|_{k_{2}}^{k_{2}} \leq \epsilon, \quad \boldsymbol{f} \geq 0
\end{aligned}
$$


where $R$ is the operator representing the quantity we desire to minimize, $\|\cdot\|_{k_{1}}$ and $\|\cdot\|_{k_{2}}$ are two given norms, and $\epsilon$ is a measure of noise. The choice of the operator $R$ depends on the goal of restoration. If $R$ is set to the identity matrix, then the goal is to find the minimum $k_{1}$-norm solution that satisfies the constraints in (2). On the other hand if $R=R_{s}^{-1 / 2} R_{n}^{1 / 2}$, where $R_{s}$ and $R_{n}$ are the covariance matrices of the signal and noise, respectively, the aim then is to minimize the noiseto-signal ratio. In this paper, however, we consider only $R$ as a variational operator, such as the Laplacian and the Total Variations. Therefore, the formulation in (2) is very general.

For the sake of conciseness, let us make the substitutions

$$
M(\boldsymbol{f})=\|R \boldsymbol{f}\|_{k_{1}}^{k_{1}} \text { and } N(\boldsymbol{f})=\|H \boldsymbol{f}-\boldsymbol{g}\|_{k_{2}}^{k_{2}}-\epsilon
$$

The optimization problem (2) can be written as follows:

$$
\begin{array}{ll}
\text { minimize } & M(\boldsymbol{f}) \\
\text { subject to } & N(\boldsymbol{f}) \leq 0, \quad \boldsymbol{f} \geq 0
\end{array}
$$

Assuming that $M(\boldsymbol{f})$ and $N(\boldsymbol{f})$ are convex functions, then (3) is a nonlinear convex program (NCP). Furthermore, if $M(\boldsymbol{f})$ and $N(\boldsymbol{f})$ are twice differentiable, then the solution of (3) satisfies the Karush-Kuhn-Tucker (KKT) conditions [8].

\subsection{Variational Inequality Problem}

In this subsection, the image restoration problem is transformed into a problem of solving a variational inequality. Let $L(\boldsymbol{f}, \lambda)$ be the Lagrangian function of (3)

$$
L(\boldsymbol{f}, \lambda)=M(\boldsymbol{f})+\lambda N(\boldsymbol{f})
$$

According to the well known saddle point theorem [9], the solution $\left(\boldsymbol{f}^{*}, \lambda^{*}\right)$, if it exists, is a saddle point, which satisfies the following inequalities:

$$
L\left(\boldsymbol{f}^{*}, \lambda\right) \leq L\left(\boldsymbol{f}^{*}, \lambda^{*}\right) \leq L\left(\boldsymbol{f}, \lambda^{*}\right), \quad \forall \boldsymbol{f} \geq 0, \lambda \geq 0 .
$$

Since $L\left(f, \lambda^{*}\right)$ is convex and admits a minimum at $f^{*}$, it follows from (5) that $f^{*}$ is a solution to the NCP (3) if and only if there exists a $\lambda^{*} \geq 0$ such that

$$
\begin{array}{r}
\left(\boldsymbol{f}-\boldsymbol{f}^{*}\right)^{T}\left[\nabla M\left(\boldsymbol{f}^{*}\right)+\nabla N\left(\boldsymbol{f}^{*}\right) \lambda^{*}\right] \geq 0, \quad \forall \boldsymbol{f} \geq 0 \\
\left(\lambda^{*}-\lambda\right) N\left(\boldsymbol{f}^{*}\right) \geq 0, \quad \forall \lambda \geq 0 .
\end{array}
$$

Equation (6) defines a variational inequality problem (VIP). It is well known from the projection theorem [9] that the solution of the VIP (6) satisfies the relation

$$
\begin{array}{r}
{\left[\boldsymbol{f}^{*}-\alpha_{1}\left(\nabla M\left(\boldsymbol{f}^{*}\right)+\nabla N\left(\boldsymbol{f}^{*}\right) \lambda^{*}\right)\right]^{+}-\boldsymbol{f}^{*}=0} \\
{\left[\lambda^{*}+\alpha_{2} N\left(\boldsymbol{f}^{*}\right)\right]^{+}-\lambda^{*}=0,}
\end{array}
$$

where $\alpha_{1}$ and $\alpha_{2}$ are positive constants, and [. $]^{+}$denotes projection onto the feasible region, here $[x]^{+}=\max (x, 0)$. The advantage of this formulation is that both the restored image and the regularization parameter $\lambda$ can be obtained simultaneously by solving (7), which satisfies the original NCP (3).

\subsection{A Dynamic System Formulation}

The solution to the projection formulation in (7) can be solved using a dynamic system approach. Consider the nonlinear system governed by the ordinary differential equations:

$$
\begin{aligned}
\frac{d \boldsymbol{f}}{d t} & =\left[\boldsymbol{f}-\alpha_{1}(\nabla M(\boldsymbol{f})+\nabla N(\boldsymbol{f}) \lambda)\right]^{+}-\boldsymbol{f}, \\
\frac{d \lambda}{d t} & =\left[\lambda+\alpha_{2} N(\boldsymbol{f})\right]^{+}-\lambda .
\end{aligned}
$$

It can easily be shown that the equilibrium point of (8) satisfies the conditions in (7). Therefore, provided the system is stable and reaches its equilibrium point, the solution of (7) is obtained when the the system reaches steady-state. We should note that an approximate solution can be obtained close to the equilibrium point, i.e., before the system reaches steady-state.

If the amount of noise is small, then the optimum value of $\lambda$ should be large. As the value of noise approaches zero, the optimum value of $\lambda$ tends to infinity. However, for very large values of $\lambda$ the dynamical system becomes stiff (or ill conditioned), and hence a numerical solution may diverge. To overcome this problem, we make the following substitution:

$$
\lambda=\mu /(1-\mu), \text { for } \lambda \geq 0,0 \leq \mu \leq 1 .
$$

Furthermore, if we set $\alpha_{1}=\alpha_{3}(1-\mu)$ and $\alpha_{2}=\alpha_{4} /(1-\mu)$, then the equations governing the system dynamics become

$$
\begin{aligned}
\frac{d \boldsymbol{f}}{d t} & =\left[\boldsymbol{f}-\alpha_{3}(\nabla M(\boldsymbol{f})(1-\mu)+\nabla N(\boldsymbol{f}) \mu)\right]^{+}-\boldsymbol{f} \\
\frac{d \mu}{d t} & =\left[\mu+\alpha_{4} N(\boldsymbol{f})\right]^{+}-\mu
\end{aligned}
$$

The dynamic system in (9) is better conditioned, and therefore, its numerical solution is more stable. The parameters $\alpha_{3}$ and $\alpha_{4}$ can be chosen to control the rate of convergence, and thus ensure greater stability.

\subsection{Selecting the Constraint Bound - $\epsilon$}

The choice of constraint bound $\epsilon$, can have a significant effect on the solution as the system usually converges when $\epsilon=\|\boldsymbol{g}-H \boldsymbol{f}\|_{k_{2}}^{k_{2}}$. If we make the assumption that the noise has a normal distribution with zero mean and standard deviation $\sigma_{n}$, then for sufficiently large images we can say that $\|\boldsymbol{g}-H \boldsymbol{f}\|_{2}^{2} \approx n \sigma_{n}^{2}$, and $\|\boldsymbol{g}-H \boldsymbol{f}\|_{1}^{1} \approx 2 n \sigma_{n} / \sqrt{2 \pi}$, where $n$ is the number of elements in $f$. This, however, is an upper bound. For a given noise variance, smaller blurs require smaller bounds to obtain an optimal solution. Using noise removal techniques, it is possible to choose a better bound by selecting

$$
\epsilon=\left\|\boldsymbol{g}-\boldsymbol{g}_{d}\right\|_{k_{2}}^{k_{2}}
$$

where $\boldsymbol{g}_{d}$ is the denoised version of $\boldsymbol{g}$. An excellent method for noise removal is the Interscale Orthonormal Wavelet Thresholding approach [10]. 


\section{EXPERIMENTAL RESULTS}

In order to test and evaluate the performance of the proposed approach, a number of experiments were conducted. In all experiments the Improvement in Signal to Noise Ratio (ISNR) is used as a performance measure. The ISNR is defined as

$$
I S N R=10 \log _{10} \frac{\left\|\boldsymbol{g}-\boldsymbol{f}_{o}\right\|_{2}^{2}}{\left\|\boldsymbol{f}-\boldsymbol{f}_{o}\right\|_{2}^{2}} d B
$$

Each experiment is performed with three different images of size 256. The images used are the Lena, Cameraman and Shepp-Logan Phantom images. A $9 \times 9$ uniform blur is used with additive zero-mean white Gaussian noise such that BSNR $\equiv 10 \log _{10}\left(\sigma_{H f}^{2} / \sigma_{n}^{2}\right)=20 d B$, where $\sigma_{H f}^{2}$ is the variance of $H \boldsymbol{f}$. A summary of the results can be found in Table 1. It will be necessary to refer to this table throughout this discussion as it details the specifics of each method. Some of the restored images obtained from these experiments are shown in Fig. 3, with corresponding Mean Squared Error (MSE) and Mean Absolute Error (MAE) values.

In order to test the versatility of our approach, we used two different norms, the $L_{1}$ and $L_{2}$ norms, and two different $R$ operators, the Laplacian and the adaptive Total Variations Quadratic Minimizer (TV-MM) [11]. The implementation of the minimizer presented in [11] is achieved by setting

$$
R^{T} R=D^{T} W^{(t)} D
$$

For further details on how to choose $D$ and $W^{(t)}$, the reader is referred to [11].

\section{Test 1 - Adaptive Regularization}

In the first set of experiments the ability of the proposed method to adapt to different images and levels of noise was tested. This was achieved by using the proposed method (Dyn-1) to restore the cameraman image with different levels of noise. The results are presented in Fig. 1. The results show that as the level of noise decreases the constraint is enforced more strictly and thus $\mu$ approaches 1 .

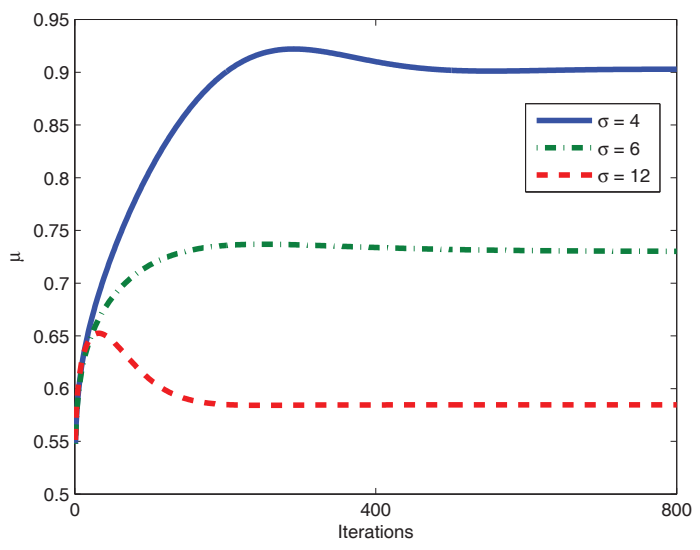

Fig. 1. Evolution of $\mu$ for different levels of noise.
In the next experiment, the same blur and the same level of noise $\left(\sigma_{n}=6\right)$ are applied to three different images. The results are presented Fig. 2, which shows that the optimum value of the regularization parameter, $\mu$, depends on the image being restored.

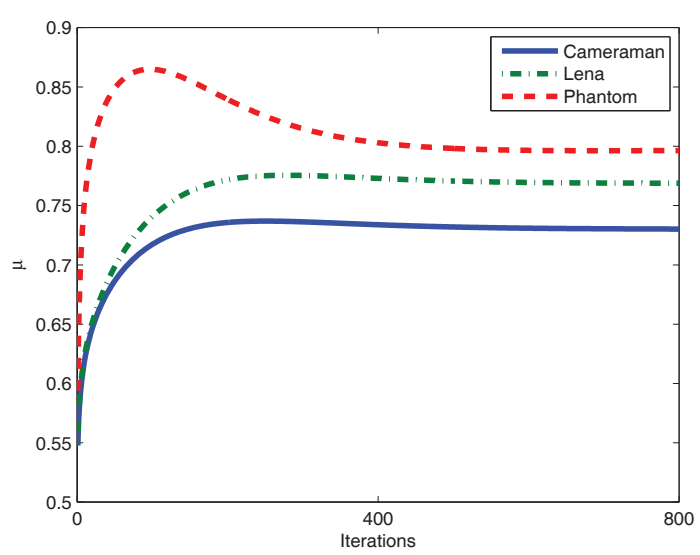

Fig. 2. Adaptation of $\mu$ for different images.

\section{Test 2 - Using Different Norms}

Using the TV-MM minimizer and (10), the effect of using different norms in the constraint was tested using the $L_{2}$ (Dyn- 1 ) and $L_{1}$ (Dyn-2) norms. The results in Table 1 show that both norms can be used successfully, with the $L_{2}$ norm providing the best results in terms of ISNR. Fig. 3 shows these results visually with the MSE and MAE reflecting the advantage of using each norm.

\section{Test 3 - Constraint Bound Selection}

In order to test the effect of bound selection the experiments in Test 2 were repeated, however this time $\epsilon$ was chosen such that $\epsilon=n \sigma_{n}^{2}$ for the $L_{2}$ norm (Dyn-3) and $\epsilon=n \sigma_{n} \frac{2}{\sqrt{2 \pi}}$ for the $L_{1}$ norm (Dyn-4). The results in Table 1 suggest that better results are obtained when noise estimation techniques from (10) are used.

\section{Test 4 - Selection of Operator $R$}

For this test the Laplacian operator was used as the variational operator to be minimized (Dyn-5). While this method performed well, the TV-MM minimizer produced significantly better results in all tests.

\section{Test 5 - Comparison with state-of-the-art Techniques}

Finally, the performance of the proposed method is compared with the methods presented in [1] and [11]. The results are summarized in Table 1, which clearly shows the proposed method achieves comparable or better results; achieving best results on the smoother Cameraman and Shepp-Logan images. 
Table 1. Summary of experimental results.

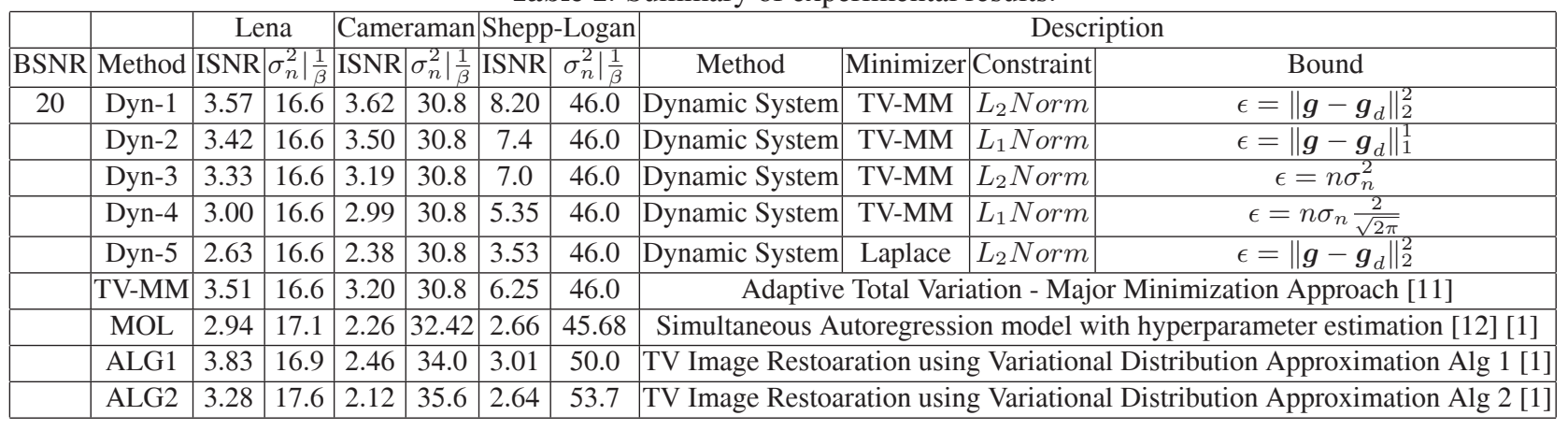

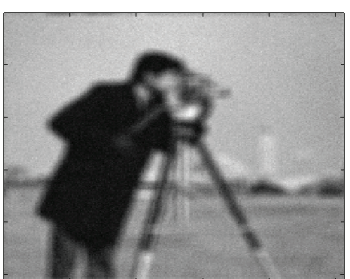

(a) Degraded Image, MSE=574.6 MAE=14.31

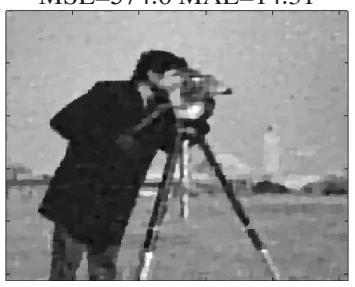

(c) Dyn-2, TV-MM L1 $\mathrm{MSE}=256.9 \mathrm{MAE}=8.77$

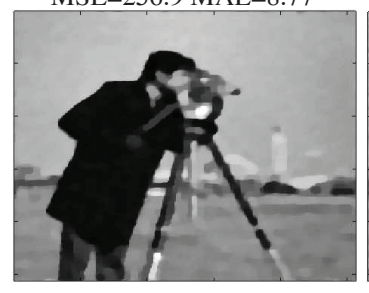

(e) Dyn-4, TV-MM L1 $\mathrm{MSE}=288.8 \mathrm{MAE}=8.62$

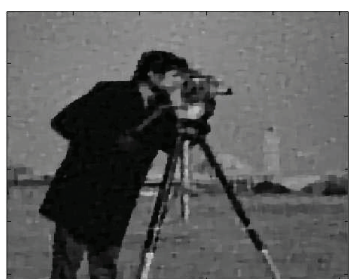

(b) Dyn-1, TV-MM L2 MSE $=249.4 \mathrm{MAE}=9.3$

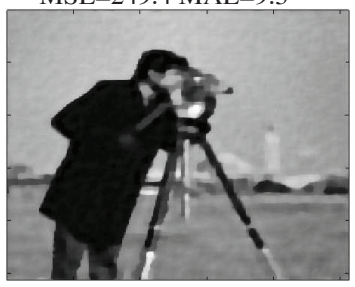

(d) Dyn-3, TV-MM L2 MSE $=363.2 \mathrm{MAE}=10.3$

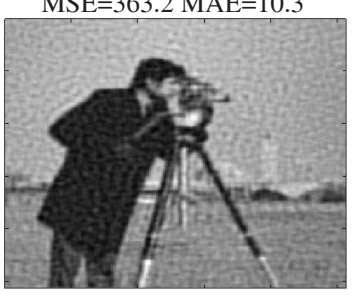

(f) Dyn-5 Laplace L2 MSE $=332.0$ MAE $=12.3$

Fig. 3. Camerman, $9 \mathrm{x} 9$ uniform blur, $\mathrm{BSNR}=20 \mathrm{~dB}, \sigma_{n}^{2}=30.8$

\section{CONCLUSION}

The method proposed in this paper uses Dynamic Systems theory to simultaneously calculate a solution to the regularized image restoration problem, in addition to a value for the regularization parameter itself. The experimental results show that using noise estimates, the proposed approach produces a good trade off between noise suppression and maintaining image fidelity. In addition, the general nature of this approach allows different norms to be implemented if required.

\section{Acknowledgment}

This work is supported in part by a grant from the Australian Research Council (ARC).

\section{REFERENCES}

[1] S. D. Babacan, R. Molina, and A. K. Katsaggelos, "Parameter estimation in tv image restoration using variational distribution approximation," IEEE Trans. Image Processing, vol. 17, no. 3, pp. 326-339, 2008.

[2] P. L. Combettes and J. C. Pesquet, "Image restoration subject to a total variation constraint," IEEE Trans. Image Processing, vol. 13, no. 9, pp. 1213-1222, 2004.

[3] P. L. Combettes and J. C. Pesquet, "Image deconvolution with total variation bounds," in Proc. 7th Int. Symp. Signal Processing and Its Applications, 2003, vol. 1, pp. 441-444 vol.1.

[4] P. Rodriguez and B. Wohlberg, "Efficient minimization method for a generalized total variation functional," IEEE Trans. Image Processing, vol. 18, no. 2, pp. 322-332, 2009.

[5] B. Wohlberg and P. Rodriguez, "An iteratively reweighted norm algorithm for minimization of total variation functionals," IEEE Signal Processing Letters, vol. 14, no. 12, pp. 948951, 2007.

[6] B. Wohlberg and P. Rodriguez, "An 11-tv algorithm for deconvolution with salt and pepper noise," in Proc. IEEE Int. Conf. Acoustics, Speech and Signal Processing, 2009, pp. 12571260.

[7] Hong Min-Cheol, T. Stathaki, and A. K. Katsaggelos, "A mixed norm image restoration," in Proc. Int. Conf. Image Processing, 1997, vol. 1, pp. 385-388 vol.1.

[8] Evar D. Nering and Albert W. Tucker, Linear programs and related problems, Computer science and scientific computing. Academic Press, Boston, 1993.

[9] David Kinderlehrer and Guido Stampacchia, An introduction to variational inequalities and their applications, Academic Press, New York, 1980.

[10] F. Luisier, T. Blu, and M. Unser, "A new sure approach to image denoising: Interscale orthonormal wavelet thresholding," IEEE Trans. Image Processing, vol. 16, no. 3, pp. 593-606, 2007.

[11] Joao P. Oliveira, Jose M. Bioucas-Dias, and Mario A. T. Figueiredo, "Adaptive total variation image deblurring: A majorization-minimization approach," Signal Processing, vol. 89, no. 9, pp. 1683-1693, 2009.

[12] R. Molina, "On the hierarchical bayesian approach to image restoration: applications to astronomical images," IEEE Trans. Pattern Analysis and Machine Intelligence, vol. 16, no. 11, pp. 1122-1128, 1994. 